\title{
Effect of thermal-treatment sequence on sound absorbing and mechanical properties of porous sound-absorbing/thermal-insulating composites
}

\author{
Chen-Hung Huang ${ }^{1}$, Cherng-Shiuan Tsay ${ }^{1}$, Ching-Wen Lou ${ }^{2, b^{*}}$, Yu-Chun Chuang ${ }^{3}$, \\ Ying-Huei Shih ${ }^{3}$ and Jia-Horng $\operatorname{Lin}^{3,4,5, a^{*}}$ \\ ${ }^{1}$ Department of Aerospace and Systems Engineering, Feng Chia University, Taichung City \\ 40724,Taiwan, R.O.C.
}

${ }^{2}$ Institute of Biomedical Engineering and Materials Science, Central Taiwan University of Science and Technology, Taichung 40601, Taiwan, R.O.C..

${ }^{3}$ Laboratory of Fiber Application and Manufacturing, Department of Fiber and Composite Materials, Feng Chia University, Taichung City 40724, Taiwan, R.O.C..

${ }^{4}$ School of Chinese Medicine, China Medical University, Taichung City 40402, Taiwan.

${ }^{5}$ Department of Fashion Design, Asia University, Taichung City 41354, Taiwan, R.O.C..

ajhlin@fcu.edu.tw, ${ }^{\text {c }}$ cwlou@ctust.edu.tw

\begin{abstract}
Due to recent rapid commercial and industrial development, mechanical equipment is supplemented massively in the factory and thus mechanical operation causes noise which distresses living at home. In livelihood, neighborhood, transportation equipment, jobsite construction noises impact on quality of life not only factory noise. This study aims to preparation technique and property evaluation of porous sound-absorbing/thermal-insulating composites. Hollow threedimensional crimp PET fibers blended with low-melting PET fibers were fabricated into hollow PET/low-melting PET nonwoven after opening, blending, carding, lapping and needle-bonding process. Then, hollow PET/low-melting PET nonwovens were laminated into sound-absorbing/thermal-insulating composites by changing sequence of needle-bonding and thermal-treatment. The optimal thermal-treated sequence was found by tensile strength, tearing strength, sound-absorbing coefficient and thermal conductivity coefficient tests of porous composites.
\end{abstract}

\section{Introduction}

In the recent years, science and technology progresses increasingly, people has proposed higher requirement for living standard. Relatively, many pollution distresses such as air

* Corresponding author:cwlou@ctust.edu.tw 
pollution, noise pollution, and water pollution occur leading to livelihood trouble and environment burden [1-2]. Noise pollution is of the deepest feeling to impact human life. Compared to pollutions from other matter, noise pollution becomes easily ameliorative, but it differs followed with living environment and personal sensitivity and brings diversified influences. Vibration and noise are the two sides of the same sound, and vibration produces noise, and also noise triggers the vibration of construction. When sound wave exceeds the administered value and feels bored, it becomes noise. Excessive noise harms human mentality and physiology [3-5].

In this study, hollow three- dimensional crimp PET fibers blended with low-melting PET fibers were fabricated into hollow PET/low-melting PET nonwoven after opening, blending, carding, lapping and needle-bonding process. Then, hollow PET/low-melting PET nonwovens were laminated into sound-absorbing/thermal-insulating composites by changing sequence of needle-bonding and thermal-treatment. The optimal thermal-treated sequence was found by tensile strength, tearing strength, sound-absorbing coefficient and thermal conductivity coefficient tests of porous composites. This research achievement can provide a new developing technique of industrial high acoustic-absorptivity composite boards for textiles and aerospace industries.

\section{Experimental}

\subsection{Materials}

7 D hollow crimped PET staple fiber (PET, length: $64 \mathrm{~mm}$, manufactured by Far Eastern New Century Corporation, Taiwan) and 4 D low-melting point PET fiber (LPET, length: $51 \mathrm{~mm}$, manufactured by Far Eastern New Century Corporation, Taiwan). Sample Preparation. PET fibers were blended with different blending ratio of LPET fibers. The blending ratio of these two fibers was $7: 3 \mathrm{wt} \%$ after formability and sound-absorbing evaluations. During thermal treatment, the processing sequences were changed, ex. needle bonding-then-hot pressing $(\mathrm{NH})$, hot pressing-then-needle bonding $(\mathrm{HN})$ and merely hot-pressing $(\mathrm{H})$. Effect of thermal-treated processing sequence on porous sound-absorbing/thermal-insulating composites was evaluated by measurements of tensile strength, tearing strength, sound-absorbing coefficient and thermal conductivity coefficient.

\subsection{Measurements}

\subsection{Tensile Strength}

This test uses a specified tensile speed of $300 \mathrm{~mm} / \mathrm{min}$, and follows ASTM D5035-11. Ten samples of each specification are taken, samples are sized as $180 \mathrm{~mm} \times 25.4 \mathrm{~mm}$, the distance between clamps is $75 \mathrm{~mm}$.

\subsection{Tearing Strength}

Ten samples of each specification are tested for tearing strength as specified in ASTM D5035-06. The test speed is $300 \mathrm{~mm} / \mathrm{min}$, the distance between clamps is $25.4 \mathrm{~mm}$, the depth of the incision is $15 \mathrm{~mm}$, and samples have a size of $75 \mathrm{~mm} \times 150 \mathrm{~mm}$. 


\subsection{Acoustic Absorption Coefficient}

As specified in ASTM E1050-12, a two-microphone impedance tube (Automotive Research \& Testing Center, Taiwan) measures the acoustic absorption coefficient of the PU foam and fiber-reinforced PU foam composite boards at a frequency between 125 and 4000 Hz. Samples are cut into circular sections with a diameter of $38 \mathrm{~mm}$.

\subsection{Thermal Conductivity}

Thermal conductivity test is performed on the PU foam and fiber-reinforced PU foam composite boards at $100{ }^{\circ} \mathrm{C}$ as specified in ASTM C177-10, with a Guarded-Hot-Plate Apparatus (DRX-I-SPB, Xiangtan Huafeng Equipment Manufacture Co. Ltd., China). The size of samples is $15 \mathrm{~cm} \times 15 \mathrm{~cm} \times 2 \mathrm{~cm}$. Three samples of each specification are tested for the mean.

\section{Results and Discussion}

\subsection{Tensile Strength}

This part discusses effect of thermal-treated sequence $(\mathrm{NH}, \mathrm{HN}$ and $\mathrm{H})$ on tensile strength and elongation of porous sound-absorbing/thermal-insulating composites. Figure 1(a) shows that sample along CD orientation after hot pressing had the optimum tensile strength. The reason is that after needle bonding, fibers adjacent needle-punched opening pushed peripherad forming some bigger holes which cannot effectively transmit and disperse stress when it is stressed due to lack of fibers. Figure 1(b) shows that tensile elongation of porous composites. It is found that processing change affected tensile elongation not obviously. According to tensile strength result, composites by hot-pressing had optimum tensile strength, as high as $749.3 \mathrm{~N}$ along CD.

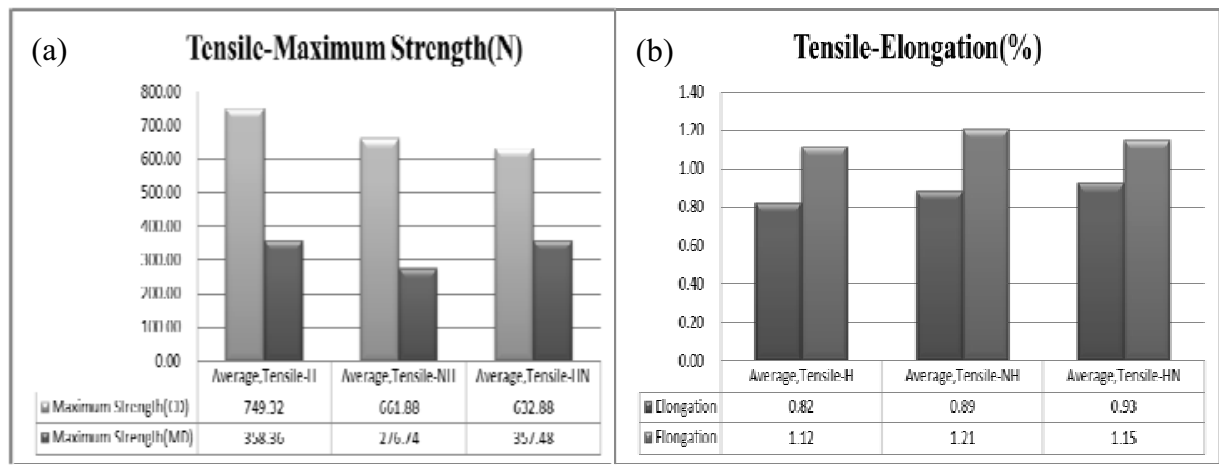

Figure 1. Tensile strength (a) and tensile elongation (b) of PET/LPET composites with different thermal-treated sequences.

\subsection{Tearing Strength}

Figure 2 shows tearing strength and elongation of porous sound-absorbing / thermal-insulating composites with different thermal-treated sequence. It is found that hot-pressed composite has the optimal tear strength along CD. The reason is the same as 
tensile strength. Compared $\mathrm{HN}$ and $\mathrm{NH}$ composites, the former had the higher tear strength, which is because for $\mathrm{NH}$ composite processed by needle bonding and then hot pressing, adjacent fibers to needle points easily formed thermal bonding spots leading to maldistribution of thermal-bonding points for the whole composites; while for HN sample that was firstly hot-pressed and then needle-boned, thermal-bonding points distributed uniform not facile to stress concentration. Figure 2(b) shows that tear elongation of porous sound-absorbing / thermal-insulating composites. It is found that processing change impacted on tear elongation insignificantly. Therefore, testing result displays that sample after hot pressing had the maximum tear strength along CD, reaching $1583.3 \mathrm{~N}$.

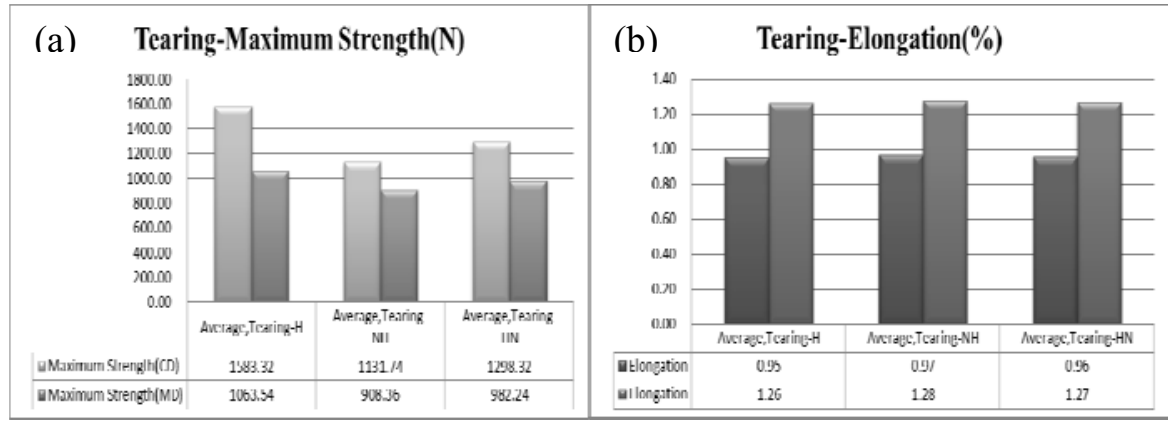

Figure 2. Tearing strength (a) and tearing elongation (b) of PET/LPET composites with different thermal-treated sequences.

\subsection{Acoustic Absorption Coefficient}

Recent reference showed noise frequency was $1000 \mathrm{~Hz}$ at experessway [6], therefore, acoustic absorption coefficient at $1000 \mathrm{~Hz}$ of porous composites was evaluated in Figure 3(a). For only hot-pressed $(\mathrm{H})$ sample, bulky nonwoven via hot pressing produced merely melting, and melting fibers formed sheet-bonding on the surface which improved acoustic resistance but being incident into sample difficultly and thus resulted in finite sound absorption. While for $\mathrm{HN}$ sample, because hot pressing makes the structure compact, and sound-absorbing effect is not good even after needle-bonding. But NH sample had the optimum sound absorption coefficient at $1000 \mathrm{~Hz}$, because higher amount of sound waves can be incident into holes of samples which further increased friction loss and reflection loss between sample and sound waves. Figure 3(b) shows that at $4000 \mathrm{~Hz}$ frequency, NH sample had the optimum sound absorption coefficient. Therefore, porous sound-absorbing / thermal-insulating composite can be processed by first needle bonding and then hot pressing.

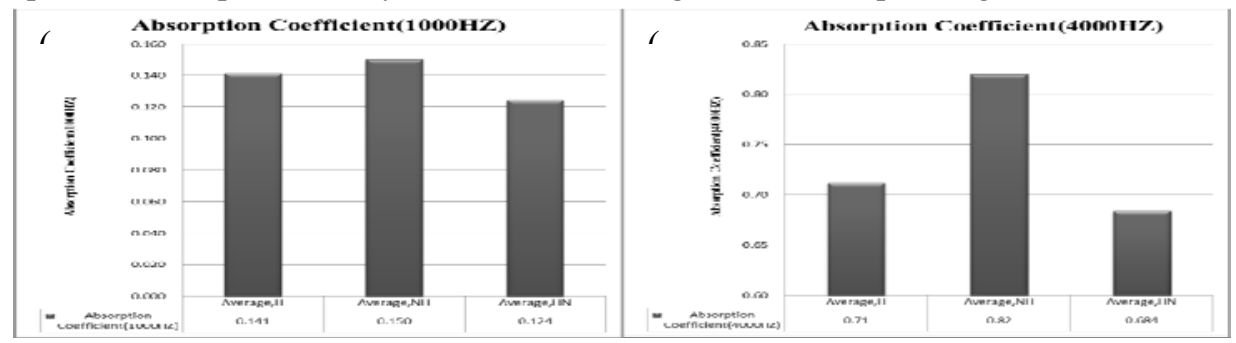

Figure 3. Acoustic Absorption Coefficient of PET/LPET composites with different thermal-treated sequences at frequency of (a) $1000 \mathrm{~Hz}$; and (b) $4000 \mathrm{~Hz}$. 


\subsection{Thermal Conductivity}

Figure 4 shows thermal conductivity of porous sound-absorbing / thermal-insulating composites with different thermal-treated sequence. It displays that thermal-treated sequence does not affect the thermal conductivity significantly. Whatever $\mathrm{HN}, \mathrm{NH}$ and $\mathrm{H}$ samples, the thermal conductivity was $0.14 \sim 0.16 \mathrm{~W} / \mathrm{mK}$. This is because regardless of processing design, composite is a still porous fibrous board, and its irregular fiber arrangement effectively hindered the air flow in addition to retention air in fiber pores which makes sample having better thermal insulating and prevention effects. NH sample had the lowest thermal conductivity of $0.14 \mathrm{~W} / \mathrm{mK}$, showing the optimal thermal-insulating effect.

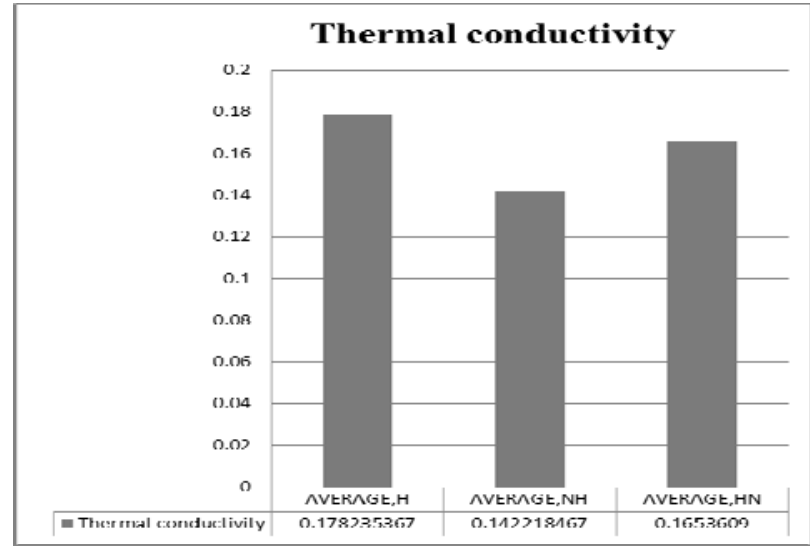

Figure 4. Thermal Conductivity of PET/LPET composites with different thermal- treated sequences.

\section{Conclusion}

Porous sound-absorbing/thermal-insulating composites in this study has characteristic of easy construction and light weight, and can be appropriate for improving sound-absorbing/thermal-insulating effect as vehicle sound absorbing material, building decoration material, aircraft cabin. According to tests, it shows that sample processed by needle bonding and then hot pressing ( $\mathrm{NH}$ sample) has the optimum sound absorbing and thermal insulating effects, and its sound absorption coefficient reaches 0.8 at $4000 \mathrm{~Hz}$ and thermal conductivity is $0.14 \mathrm{~W} / \mathrm{mK}$.

\section{Acknowledgements}

The authors would like to thank Ministry of Science and Technology of Taiwan, for financially supporting this research under Contract MOST 104-2622-E-035-023-CC2.

\section{References}

1. T.T. Li, Y.C. Chuang, C.H. Huang, C.W. Lou, J.H. Lin.Applying Vermiculite and Perlite Fillers to Sound-Absorbing/Thermal-Insulating Resilient PU Foam Composites. Fiber Polym. 16(3) (2015) 691-698.

2. C.H. Huang, J.H. Lin, and Y.C. Chuang, Manufacturing process and property evaluation of sound-absorbing and thermal-insulating polyester fiber/polypropylene /thermoplastic polyurethane composite board, J Ind Text. 43(4) (2014) 627-640. 
3. Y. Nakai and H. Masutani: Noise-Induced Vasoconstriction in the Cochlea. Acta Oto-Laryngol. 47 (1988) 23.

4. L. Thiery and B.C. Meyer: Hearing Loss Due to Partly Impulsive Industrial; Noise Exposure at Levels Between 87 and 90 dB(A). J. Acoust. Soc. Am. 84 (1988) 651.

5. R.S. Yan, R. Wang, C.W. Lou, and J.H. Lin. Manufacturing Technique and Acoustic Evaluation of Sandwich Laminates Reinforced High-resilience Inter/Intra-ply Hybrid Composites. Fiber Polym. 15(10) (2014) 2201-2210. 